\title{
The application of Kohonen Self-Organizing Maps for the classification of the electronic components and reliability improvement of onboard equipment
}

\author{
R O Mishanov ${ }^{1}$ \\ ${ }^{1}$ Samara National Research University, Moskovskoe shosse 34, Samara, Russia, 443086
}

\begin{abstract}
The technique of the electronic products classification into classes of the acceptable and potentially unacceptable instances using Kohonen Self-Organizing Maps (SOM) is given. The methodology was tested on two samples of special-purpose electronic components using application software. The analysis of the SOM is given. The classification accuracy is estimated and a comparison table, that includes the results of cluster analysis, is given. The recommendations for improving the classification quality are developed.
\end{abstract}

\section{Introduction}

The development and perform actions for the improving reliability of the radioelectronic equipment, installed on the spacecraft, is one of the most important tasks assigned to scientists and specialists in the space industry. The task of increasing reliability is most successfully solving by forecasting the future state of equipment [1]. The search for the new forecasting methods, based on the use of mathematical models and most applicable to non-renewable equipment, is relevant at this stage in the development of science and technology [2]. Such methods include the individual forecasting (IF). Its main idea is that the value of the informative parameter or the results of monitoring each instance using the forecast model formulates a conclusion about the potential reliability of this instance [1].

In paper [3] forecast models are presented for the CMOS chips and zener diodes (stabilitrons) samples using regression models. Verification of the results was carried out using the method of discriminant functions. The methods allowing to split the initial samples of electronic components into classes of reliable and potentially unreliable elements for verifying the results of the IF are presented in works [4, 5]. These methods are based on the cluster analysis using the k-means clustering and based on the agglomerate methods of hierarchical clustering with subsequent evaluation of the results.

This paper discusses the possibility of using Self-Organizing Maps (SOM) to classify electronic components into classes of reliable and potentially unreliable instances. Furthermore, the technique of using such network to classify samples of electronic components is presented.

\section{Kohonen networks}

The Kohonen networks are referred to as self-organizing neural networks, which allow to identify groups of input data vectors with similar properties [6,7].

The Kohonen network is a single-layer network constructed from WTA neurons (Winner Takes All). Figure 1 shows the structure of such network. 


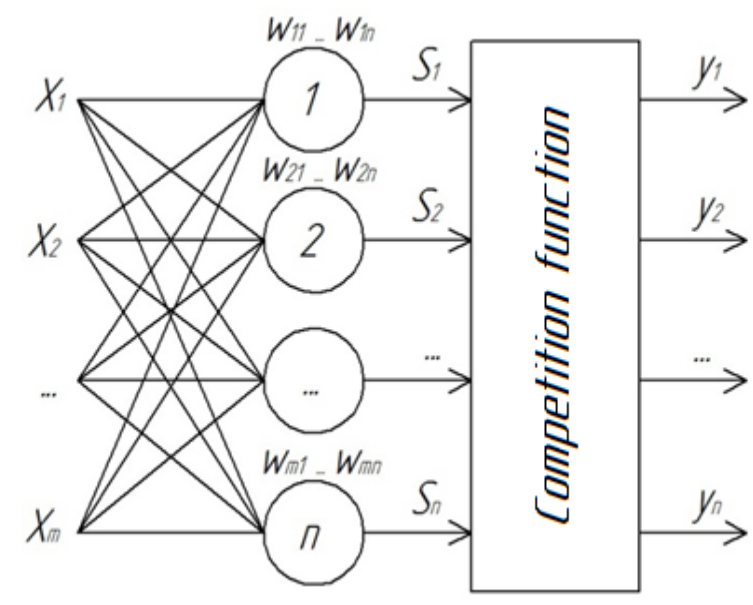

Figure 1. A structure of the Kohonen network.

Each neuron is associated with each component of the input data $x_{1}, x_{2}, \ldots, x_{m}$ (the input data vector), which in this case is a set of informative parameters for each instance of the sample. Each neuron is presented as a linear combiner:

$$
S_{j}=b_{j}+\sum_{i=1}^{m} w_{i j} x_{i}
$$

where $S_{i}$ - is an output result of the combiner; $j$ - is a neuron number; $i-$ is a number of the informative parameter; $b_{i}$ - is a threshold; $w_{i j}$ - is a weight of the $\mathrm{i}^{\text {th }}$ parameter of the $\mathrm{j}^{\text {th }}$ neuron.

From the output of each neuron the result goes to the competition function, which defines the neuron with the maximum result value at the output and assigns it a value of one. The remaining output signals are assigned a value of zero. For the neuron-winner, the following condition is fulfilled:

$$
d\left(x, w_{j}\right)=\min _{1 \leq i \leq n} d\left(x, w_{i}\right),
$$

whered $(x, w)$ - is a distance between the vectors of the input data and the vector of the synaptic weights, $j$ - is a number of the neuron-winner. It should be noted that the distance in the selected metric is an Euclidean distance.

The neuron-winner is a neuron, the vector of synaptic weights $w_{i}$ of which differs least from the vector of input data. In the case of determining the maximum result at the outputs of several neurons, the result equal to one is assigned to one of them, and the outputs of the remaining neurons are assigned a value of zero. Neuron-winner defines such a group, to which the vector of input data is closest .The scales are adjusted each training cycle:

$$
w_{j}^{(k+1)}=w_{j}^{(k)}+\mathrm{n}_{j}^{(k)}\left[X-w_{j}^{(k)}\right],
$$

where - is a number of the training cycle; $X$ - is an input vector; $\eta_{i}^{(k)}-$ is a coefficient of learning rate of the $\mathrm{j}^{\text {th }}$ neuron in the $\mathrm{k}^{\text {th }}$ cycle.

Thus, the neuron, whose synaptic weights vector was closer to the vector of the input data, is corrected and becomes even closer. Also, the weights of the nearest neurons are corrected to the neuron-winner. Thus, the probability of this neuron to become a neuron-winner is increased when a closer vector of data is input.

The training of the Kohonen network consists in the selection of weight values when errors are minimized.

\section{Classification technique}

The developed classification technique based on Kohonen self-organizing maps is as follows:

- determination of the vector of input data based on the results of the learning experiment;

- definition of network construction parameters;

- analysis of the results with an assessment of the accuracy of the classification. 
Two samples of CMOS chips, obtained in [3-5], were chosen as the study samples. The volume of both samples is 50 instances. Time delay on the leading edge of the signal $x_{1}\left(\mathrm{t}_{\mathrm{p}}^{+}, \mu \mathrm{s}\right)$ and the critical supply voltage $x_{2}\left(\mathrm{~V}_{\mathrm{cs}}, \mathrm{V}\right)$ are used as the informative parameters. The leakage current drift $y$ is assumed as the forecasting parameter.

Thus, the vector of input data is a set of informative parameters $x_{1}$ and $x_{2}$ of each sample.

The analytical platform "Deductor Academic 5.1" was chosen as a software tool for building Kohonen network. The following building parameters need to be defined:

- size of the training and test set;

- a network size (number of neurons);

- a recognition criterion (the amount of recognition error);

- rate and radius of learning;

- number of clusters.

Because the samples size is small, the training set should be as large as possible and sufficient for the correct building of the network. In this case, the test set should be sufficient for testing the network with $100 \%$ recognition. Based on the set conditions, the size of the training set was 45 instances $(90 \%$ of the sample), the test set size -5 instances ( $10 \%$ of the sample).

The network size is chosen in such a way as to exclude the appearance of "dead" neurons (vector of synaptic weights is significantly removed from the vector of input data). Such neurons turn out to be inactive in network training, because they cannot win competition from the nearest neurons. This situation leads to the fact that the input data is interpreted by a smaller number of neurons than was originally set, which introduces distortions into the final result.

Networks with a dimension of $5 \times 4$ neurons (cells) were chosen for testing the presented samples. The type of cells is selected hexagonal: it more accurately displays the Cartesian distance between the cell centres. The remaining parameters of the Kohonen network building are presented in table 1 .

Table 1. Kohonen network building parameters.

\begin{tabular}{ll}
\hline \multicolumn{1}{c}{ Network parameter } & \multicolumn{1}{c}{ Criterion } \\
\hline Recognition error value & $\begin{array}{l}\text { An example is considered recognized if the } \\
\text { recognition error is less than } 0,05\end{array}$ \\
& 200 \\
Number of the stages & In the beginning of the training: 0,3 \\
Training rate $\eta$ & In the end of the training: 0,005 \\
& In the beginning of the training: 2 \\
Training radius & In the end of the training: 0,2 \\
& Fixed number: 2 \\
\hline
\end{tabular}

From the whole set of variants of networks built according to the given parameters, the only option was chosen for each sample, at which the minimum average recognition error for the training set was achieved with 100\% recognition of both the training set and the test set.

Because a fixed number of clusters equals two, according to the classification table it is not difficult to determine to which class of products the network relates each instance.

To estimate the accuracy of the classification, the technique given in $[4,8]$ was applied.

\section{Results of building a network for sample №1}

Figure 2 shows the maps of the input data $x_{1}, x_{2}$ and the output data $y$ for the network. The maps of such parameters are called component planes. Figure 3 shows the density hit matrix, the matrix of distances between the centres of neurons, and also a map of cell decomposition into two clusters.

Figure 2 shows that the maps of the neuron inputs (maps X1, X2) and the output map have a similar appearance: on the left there are cells that characterize neurons with smaller weights, and on the right there are cells characterizing neurons with large weights. Because the output map of neurons (map Y) is a projection of the maps of neuron inputs, its appearance will be similar. 


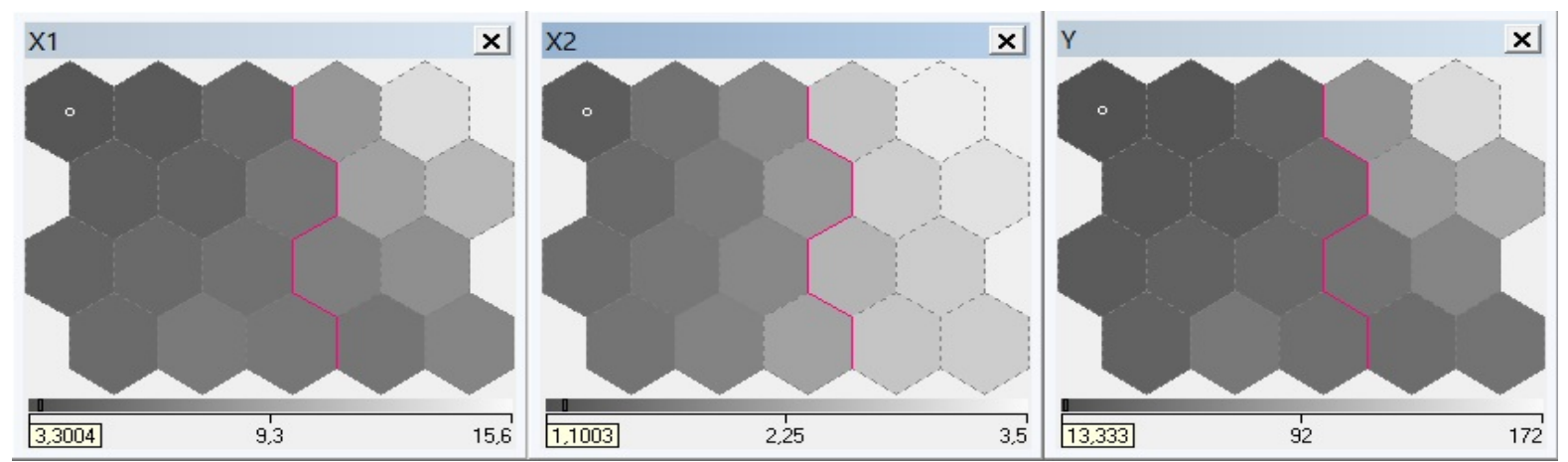

Figure 2. The maps of the input data $x_{1}, x_{2}$, output data $y$ for the sample №1.

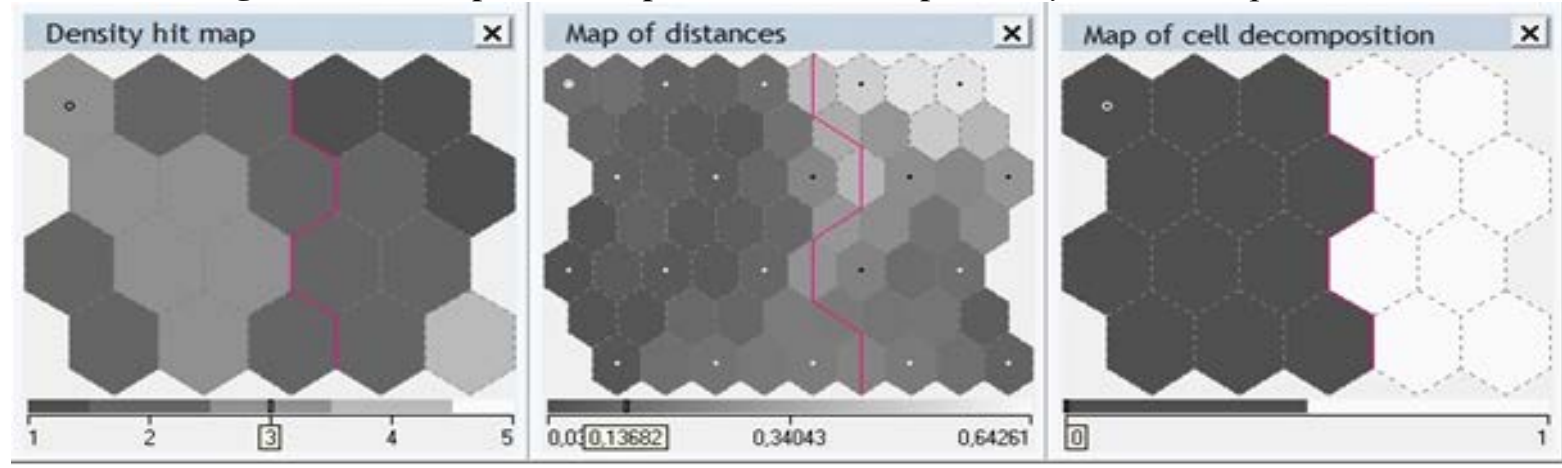

Figure 3. The density hit map, the map of distances, the map of cell decomposition for the sample №1.

Figure 3 shows that the map of cell decomposition is divided into 2 areas that characterize clusters. In this case, cluster 0 is a class of the reliable instances (class 1 ), cluster 1 is a class of the potentially unreliable instances (class 2). Table 2 provides the information on assigning each instance to a specific class, and $K_{\text {fact }}$ means the actual belonging of the instance to a class, $K_{\text {rec. }}-$ is an assignment of the instance to a class based on recognition results using Kohonen maps.

Table 2 shows that for some instances, the actual class and the recognition class do not match. Therefore, it is necessary to estimate the accuracy of this classification. For this the technique presented in $[4,8]$ may be applied. The results of the classification accuracy evaluation are presented in table 3.

Table 2. The ownership of the sample №1 instances to classes.

\begin{tabular}{ccccccccc}
\hline $\begin{array}{c}\text { № } \\
\text { instance }\end{array}$ & $K_{\text {fact }}$ & $K_{\text {rec. }}$ & $\begin{array}{c}\text { № } \\
\text { instance }\end{array}$ & $K_{\text {fact }}$ & $K_{\text {rec. }}$ & $\begin{array}{c}\text { № } \\
\text { instance }\end{array}$ & $K_{\text {fact }}$ & $K_{\text {rec. }}$ \\
\hline $\mathbf{1}$ & 1 & 1 & $\mathbf{1 8}$ & 1 & 1 & $\mathbf{3 5}$ & 2 & 2 \\
$\mathbf{2}$ & 1 & 2 & $\mathbf{1 9}$ & 1 & 1 & $\mathbf{3 6}$ & 1 & 1 \\
$\mathbf{3}$ & 1 & 1 & $\mathbf{2 0}$ & 1 & 1 & $\mathbf{3 7}$ & 1 & 1 \\
$\mathbf{4}$ & 2 & 1 & $\mathbf{2 1}$ & 1 & 1 & $\mathbf{3 8}$ & 1 & 2 \\
$\mathbf{5}$ & 1 & 1 & $\mathbf{2 2}$ & 2 & 2 & $\mathbf{3 9}$ & 2 & 2 \\
$\mathbf{6}$ & 1 & 1 & $\mathbf{2 3}$ & 1 & 1 & $\mathbf{4 0}$ & 2 & 2 \\
$\mathbf{7}$ & 2 & 1 & $\mathbf{2 4}$ & 1 & 1 & $\mathbf{4 1}$ & 1 & 1 \\
$\mathbf{8}$ & 1 & 1 & $\mathbf{2 5}$ & 2 & 2 & $\mathbf{4 2}$ & 1 & 1 \\
$\mathbf{9}$ & 1 & 2 & $\mathbf{2 6}$ & 1 & 2 & $\mathbf{4 3}$ & 2 & 2 \\
$\mathbf{1 0}$ & 1 & 1 & $\mathbf{2 7}$ & 1 & 1 & $\mathbf{4 4}$ & 2 & 2 \\
$\mathbf{1 1}$ & 1 & 1 & $\mathbf{2 8}$ & 1 & 1 & $\mathbf{4 5}$ & 1 & 1 \\
$\mathbf{1 2}$ & 1 & 1 & $\mathbf{2 9}$ & 1 & 1 & $\mathbf{4 6}$ & 2 & 2 \\
$\mathbf{1 3}$ & 1 & 1 & $\mathbf{3 0}$ & 1 & 1 & $\mathbf{4 7}$ & 2 & 2 \\
$\mathbf{1 4}$ & 2 & 1 & $\mathbf{3 1}$ & 1 & 1 & $\mathbf{4 8}$ & 1 & 1 \\
$\mathbf{1 5}$ & 2 & 2 & $\mathbf{3 2}$ & 2 & 2 & $\mathbf{4 9}$ & 1 & 1 \\
$\mathbf{1 6}$ & 1 & 2 & $\mathbf{3 3}$ & 1 & 1 & $\mathbf{5 0}$ & 1 & 1 \\
$\mathbf{1 7}$ & 1 & 2 & $\mathbf{3 4}$ & 2 & 2 & & & \\
\hline
\end{tabular}


Table 3. The results of the classification accuracy evaluation for the sample №1.

\begin{tabular}{lc} 
Criterion & Value \\
& \\
\hline Producer's risk $\left(\alpha\right.$-risk) $P_{\text {prod. }}$ & 0,333 \\
Consumer's risk $\left(\beta\right.$-risk) $P_{\text {cons. }}$ & 0,094 \\
Risk of the incorrect decision $P_{\text {inc. d. }}$ & 0,18 \\
\hline
\end{tabular}

\section{Results of building a network for sample №2}

Figure 4 shows the maps of the input data $x_{1}, x_{2}$ and the output data $y$ for the network. Figure 5 shows the density hit matrix, the matrix of distances between the centres of neurons, and also a map of cell decomposition into two clusters.
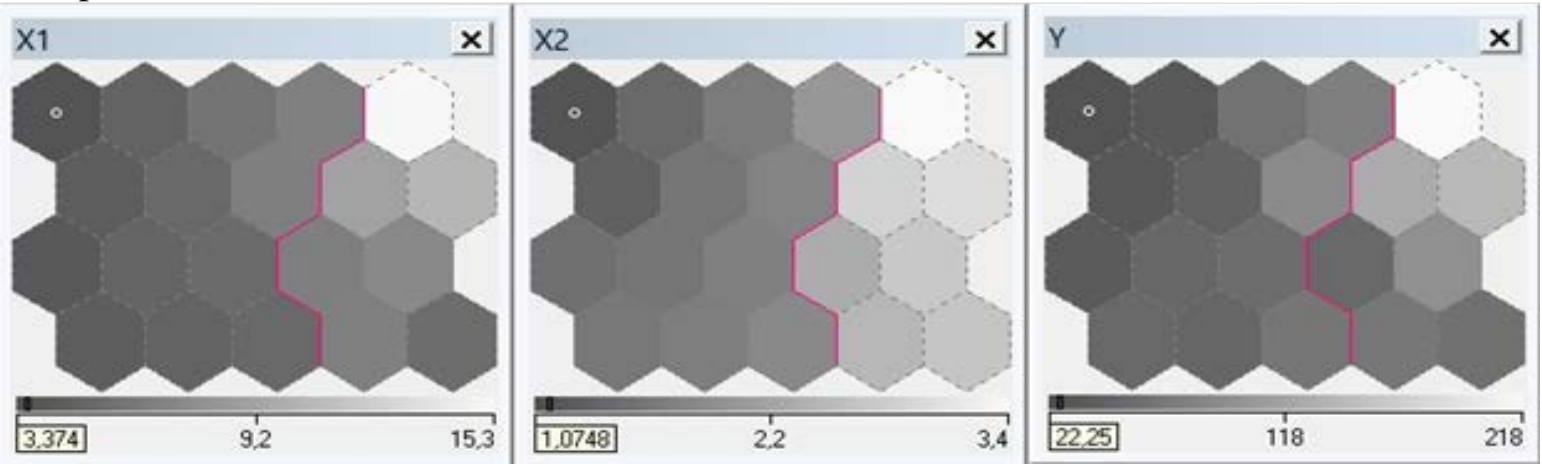

Figure 4. The maps of the input data $x_{1}, x_{2}$, output data $y$ for the sample №2.

Figure 4 shows that the neuron input maps and the output map also have a similar appearance: on the left are cells that characterize neurons with smaller weights, and on the right are cells characterizing neurons with large weights.
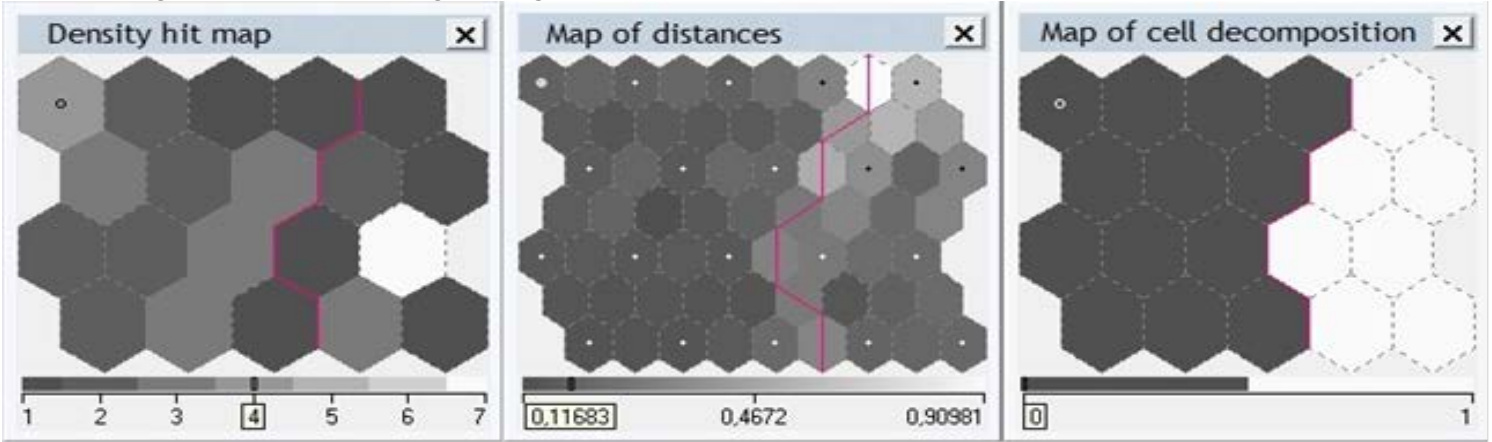

Figure 5. The density hit map, the map of distances, the map of cell decomposition for the sample №2.

Figure 5 shows that the map of cell decomposition is divided into 2 areas. Cluster 0 is a class of reliable instances (class 1 ), cluster 1 is a class of potentially unreliable instances (class 2). Table 4 provides information on assigning each instance to a specific class. Table 4 shows that for some instances, the actual class and the recognition class do not match. Therefore, it is necessary to estimate the accuracy of this classification.

Table 5 compares theaccuracy estimatesofthe classification with the best results obtained in [4].

\section{Conclusion}

The results are explained by the fact that small samples were used as input data, and, consequently, small training sets are used. The size of the networks was also limited, because with increasing network size the "dead" neurons appear, which should be avoided. One of the advantages of the developed technique is that the trained network is able to recognize any instance of the dot of products that is not part of the sample, which could not be done by cluster analysis algorithms. Thus, the 
developed technique can be recommended both as a method for verifying the results of the IF, and as the technique of the IF.

Table 4. The ownership of the sample №2 instances to classes.

\begin{tabular}{ccccccccc}
\hline $\begin{array}{c}\text { № } \\
\text { instance }\end{array}$ & $K_{\text {fact }}$ & $K_{\text {rec. }}$ & $\begin{array}{c}\text { № } \\
\text { instance }\end{array}$ & $K_{\text {fact }}$ & $K_{\text {rec. }}$ & $\begin{array}{c}\text { № } \\
\text { instance }\end{array}$ & $K_{\text {fact }}$ & $K_{\text {rec. }}$ \\
\hline $\mathbf{1}$ & 2 & 2 & $\mathbf{1 8}$ & 2 & 1 & $\mathbf{3 5}$ & 1 & 1 \\
$\mathbf{2}$ & 1 & 1 & $\mathbf{1 9}$ & 1 & 1 & $\mathbf{3 6}$ & 1 & 1 \\
$\mathbf{3}$ & 2 & 2 & $\mathbf{2 0}$ & 1 & 1 & $\mathbf{3 7}$ & 1 & 1 \\
$\mathbf{4}$ & 1 & 1 & $\mathbf{2 1}$ & 2 & 1 & $\mathbf{3 8}$ & 1 & 1 \\
$\mathbf{5}$ & 1 & 1 & $\mathbf{2 2}$ & 1 & 1 & $\mathbf{3 9}$ & 1 & 1 \\
$\mathbf{6}$ & 1 & 1 & $\mathbf{2 3}$ & 2 & 2 & $\mathbf{4 0}$ & 1 & 2 \\
$\mathbf{7}$ & 1 & 1 & $\mathbf{2 4}$ & 1 & 1 & $\mathbf{4 1}$ & 2 & 1 \\
$\mathbf{8}$ & 1 & 2 & $\mathbf{2 5}$ & 1 & 1 & $\mathbf{4 2}$ & 1 & 1 \\
$\mathbf{9}$ & 2 & 2 & $\mathbf{2 6}$ & 2 & 2 & $\mathbf{4 3}$ & 1 & 1 \\
$\mathbf{1 0}$ & 2 & 2 & $\mathbf{2 7}$ & 1 & 2 & $\mathbf{4 4}$ & 2 & 2 \\
$\mathbf{1 1}$ & 1 & 1 & $\mathbf{2 8}$ & 1 & 2 & $\mathbf{4 5}$ & 2 & 2 \\
$\mathbf{1 2}$ & 1 & 1 & $\mathbf{2 9}$ & 1 & 1 & $\mathbf{4 6}$ & 2 & 2 \\
$\mathbf{1 3}$ & 1 & 1 & $\mathbf{3 0}$ & 1 & 1 & $\mathbf{4 7}$ & 2 & 2 \\
$\mathbf{1 4}$ & 1 & 1 & $\mathbf{3 1}$ & 2 & 2 & $\mathbf{4 8}$ & 2 & 2 \\
$\mathbf{1 5}$ & 1 & 1 & $\mathbf{3 2}$ & 2 & 2 & $\mathbf{4 9}$ & 1 & 1 \\
$\mathbf{1 6}$ & 1 & 2 & $\mathbf{3 3}$ & 1 & 1 & $\mathbf{5 0}$ & 1 & 1 \\
$\mathbf{1 7}$ & 1 & 1 & $\mathbf{3 4}$ & 2 & 2 & & & \\
\hline
\end{tabular}

Table 5. Comparison table of the classification accuracy.

\begin{tabular}{lcccc}
\hline \multirow{2}{*}{ Criterion } & \multirow{2}{*}{ SOM } & \multicolumn{3}{c}{ Cluster analysis algorithms } \\
\cline { 3 - 5 } & & Var. 1 & Var. 2 & Var. 3 \\
\hline Producer's risk $\left(\alpha\right.$-risk) $P_{\text {prod. }}$ & 0,263 & 0,238 & 0,238 & 0,263 \\
Consumer's risk ( $\beta$-risk) $P_{\text {cons. }}$ & 0,097 & 0,069 & 0,034 & 0,129 \\
Risk of the incorrect decision $P_{\text {inc. d. }}$ & 0,16 & 0,14 & 0,12 & 0,18 \\
\hline
\end{tabular}

\section{References}

[1] Piganov M N 2009 Reliability prediction of electronic equipment Scientific and Technical Bulletins SPbGPU. Series "Informatics. Telecommunications. Management" 1 175-182

[2] Piganov M N, Tyulevin S V and Erantseva E S 2015 The Experience of Designing and Application of CAD Systems in Microelectronics (Lviv: IEEE) 367-371

[3] Mishanov R O, Tyulevin S V, Piganov M N and Erantseva E S 2017 Forecasting models generation of the electronic means quality Mathematical Modeling 1 124-129

[4] Mishanov R O and Piganov M N 2017 Method of verification of the results of individual forecasting of products of space radio-electronic equipment with the help of algorithms of cluster analysis Physics of wave processes and radio engineering systems 20 55-63

[5] Mishanov R O 2017 Method of applying cluster analysis for the classification of electronic products and improving the reliability of equipment Izvestiya of the Samara Scientific Center RAS 19 414-419

[6] Kohonen T 2008 Self-organizing maps (Moscow: BINOM Laboratoriya znanij) p 655

[7] Haykin S 2006 Neural networks: full course (Moscow: Vilyams) p 1104

[8] Tyulevin S V 2016 Individual forecasting of electronic means: schoolbook (Samara: Samara University) p 65 